\title{
Preparation and Characterisation of Highly Stable Iron Oxide Nanoparticles for Magnetic Resonance Imaging
}

\author{
David Kovár̆, ${ }^{1,2}$ Aneta Malá, ${ }^{3}$ Jitka Mlčochová, ${ }^{1}$ Michal Kalina, ${ }^{4}$ Zdenka Fohlerová, ${ }^{2}$ \\ Antonín Hlaváček, ${ }^{1}$ Zdeněk Farka, ${ }^{1}$ Petr Skládal, ${ }^{1}$ Zenon Starčuk Jr., ${ }^{3}$ Radovan Jiřík, ${ }^{3}$ \\ Ondřej Slabý, ${ }^{1}$ and Jaromír Hubálek ${ }^{2}$ \\ ${ }^{1}$ Central European Institute of Technology, Masaryk University, Brno, Czech Republic \\ ${ }^{2}$ Central European Institute of Technology, Brno University of Technology, Brno, Czech Republic \\ ${ }^{3}$ Institute of Scientific Instruments of the Czech Academy of Sciences, Brno, Czech Republic \\ ${ }^{4}$ Materials Research Centre, Faculty of Chemistry, Brno University of Technology, Brno, Czech Republic \\ Correspondence should be addressed to Jaromír Hubálek; hubalek@feec.vutbr.cz
}

Received 2 October 2016; Revised 29 December 2016; Accepted 16 January 2017; Published 23 February 2017

Academic Editor: Paulo Cesar Morais

Copyright (C) 2017 David Kovář et al. This is an open access article distributed under the Creative Commons Attribution License, which permits unrestricted use, distribution, and reproduction in any medium, provided the original work is properly cited.

\begin{abstract}
Magnetic nanoparticles produced using aqueous coprecipitation usually exhibit wide particle size distribution. Synthesis of small and uniform magnetic nanoparticles has been the subject of extensive research over recent years. Sufficiently small superparamagnetic iron oxide nanoparticles easily permeate tissues and may enhance the contrast in magnetic resonance imaging. Furthermore, their unique small size also allows them to migrate into cells and other body compartments. To better control their synthesis, a chemical coprecipitation protocol was carefully optimised regarding the influence of the injection rate of base and incubation times. The citrate-stabilised particles were produced with a narrow average size range below $2 \mathrm{~nm}$ and excellent stability. The stability of nanoparticles was monitored by long-term measurement of zeta potentials and relaxivity. Biocompatibility was tested on the Caki-2 cells with good tolerance. The application of nanoparticles for magnetic resonance imaging (MRI) was then evaluated. The relaxivities $\left(r_{1}, r_{2}\right)$ and $r_{2} / r_{1}$ ratio calculated from MR images of prepared phantoms indicate the nanoparticles as a promising $T_{2}$-contrast probe.
\end{abstract}

\section{Background}

Magnetic resonance imaging (MRI) is a noninvasive and powerful medical imaging technique. MRI is based on nuclear magnetic resonance (NMR), which is widely used for the study of molecular structures. MR images, whose intensity reflects local relaxation rates of water protons, can be used for visual discrimination of different physiological and pathological tissues [1]. In many cases, native contrast provides sufficient anatomic and functional discrimination capability, but for specific tasks, such as characterisation of perfusion, identification of tumours, or tracking the distribution of drug carriers, the administration of a suitable potentially functionalised MRI probe, for example, a contrast agent, may be desirable. Contrast agents shorten the relaxivities $r_{1}$ and $r_{2}$ of protons of the surrounding water molecules in some ratio and, based on the mechanism of the local probewater interaction and the local concentration of the probe particles, they affect the MR image intensity. In principle, there are two basic types of contrast agent. Probes based on gadolinium $\left(\mathrm{Gd}^{3+}\right)$ act as positive $T_{1}$-contrast agents because they are paramagnetic complexes that noticeably shorten the $T_{1}$ longitudinal relaxation time and, hence, lead to signal enhancement [2]. On the contrary, iron oxide magnetic particles (IOMPs), which are the most common $T_{2}$-contrast probes, shorten the transverse relaxation time and typically result in a lower signal. Of course, relaxivity is dependent on nanoparticle (NP) size [3], surface modification [4], and the magnetic field strength used [5]. In some measurement protocols, superparamagnetic iron oxide nanoparticles (SPIOs) of sufficiently small size can serve as a positive $T_{1}$-contrast probe as well [6]. 
The IOMPs based on magnetite $\left(\mathrm{Fe}_{3} \mathrm{O}_{4}\right)$ and maghemite $\left(\gamma-\mathrm{Fe}_{2} \mathrm{O}_{3}\right)$ are popular and some products based on these materials have been approved by the US FDA for clinical medical use, for example, Sinerem (Guerbert), Clariscan (GE Healthcare), Endorem (Guebert), Resovist, Abdoscan (GE Healthcare), and Lumirem (Guebert) [7, 8]. The IOMPs are notably suitable for imaging of liver, spleen, bone marrow, and lymph nodes because the cells of reticuloendothelial systems selectively accumulate IOMPs [9]. The IOMP nanoparticles may exhibit some cytotoxicity via autoxidation and the Fenton reaction resulting in the oxidation of proteins and lipids and damage of nucleic acids [10]. The IOMPs have been found slightly cytotoxic in vitro; however, they have been shown biocompatible in vivo at low concentrations [11].

Different methods have been developed for the production of IOMP NPs [12]. A simple synthetic method not requiring special conditions is chemical coprecipitation. This reaction can be performed at $20-90^{\circ} \mathrm{C}$ and favourably realized under inert gas. The final product is obtained within minutes to a few hours. The size, shape, and composition of the magnetic NPs are dependent on the type of salts used (e.g., chlorides, sulfates, and nitrates), the molar ratio of iron ions in solution, the concentration and properties of the chosen base (typically $\mathrm{NaOH}$ or $\mathrm{NH}_{4} \mathrm{OH}$ ), the reaction temperature, the presence of a detergent, the ionic strength, and the rate of base addition. A higher mixing rate tends to decrease size and prevent aggregation. The resulting product also depends on the arrangement of the reaction, that is, whether the base is added to the iron ion solution or vice versa. This method is preferred due to its simplicity and cost effectiveness and is noted for its high yields. Significant polydispersity of the synthesised NPs is usually a disadvantage of this method. However, once the synthetic conditions are optimised and stabilised, the quality of NPs is fully reproducible as shown in this work. Sugimoto and Matijević prepared spherical magnetite by coprecipitation of ferrous ions by potassium hydroxide in the presence of nitrate [13]. One of the pioneer works reported by Massart in 1981 described an alkaline coprecipitation protocol [14]. An aqueous solution of ferrous and ferric ions in a $2: 1$ stoichiometry was mixed with ammonia solution giving gelatinous precipitate, which was isolated and further peptised. The average size of those NPs was about $12 \mathrm{~nm}$. Since that time, many other modifications have been examined. The coprecipitation of very fine maghemite NPs using citrate stabilising ions was described by Bee et al. [15].

The synthesis and surface modification of magnetic particles are limited by several aspects. According to the thermodynamic reaction, complete precipitation of $\mathrm{Fe}_{3} \mathrm{O}_{4}$ from a mixture of ferrous and ferric ions is expected within a $\mathrm{pH}$ range of 9-14 and under nonoxidising atmosphere [16]. High surface energy of particles leads to aggregation; chemical reactivity (mainly oxidation) may cause the loss of magnetic properties and dispersibility in solution. Therefore, the magnetic core can be protected by a functional shell of various properties and chemistries allowing chemical steric stabilisation and bioconjugation. Protective shells include hydrophilic nature macromolecular polymers (agarose [17], dextran [18], chitosan [19], and gelatine [20]), polymers (polyethylene glycol [21], polyethyleneimine, polyvinyl alcohol [22], and polystyrene [23]), and self-assembled (mono)layers (silica and phospholipids [24]). The van der Waals interactions in this case become negligible. Electrostatic repulsion helps stabilise the dispersion of NPs in solution, which is usually given by electric charges introduced at the NPs' surface coming from adsorption of charged ions or ionisation of hydroxyl groups at the IOMPs. The electric surface charge appearing on the surface is affected by ionic strength and $\mathrm{pH}$. This behaviour is remarkable for coating species that are able to gain or lose protons (citric acid/citrate, orthophosphoric acid/phosphate, oleic acid, etc.) $[15,25]$. Another widely used technique is controllable encapsulation of NPs in a silica shell. The silica layer helps protect the magnetic core against oxidation and sintering and, thus, prevents the aggregation of NPs [26]. The pH stability of silica and silanised NPs is given by the isoelectric point of silica close to $\mathrm{pH} 2$ [27]. For in vivo applications, the stabilisers should be biocompatible and biodegradable. They can be incorporated to the NPs during their synthesis or in a postsynthesis manner.

The chemical coprecipitation of citrate-stabilised NPs is described here. NP size was analysed by atomic force microscopy (AFM) and dynamic light scattering (DLS). The zeta potential was monitored over a long period and the properties of the NPs important for MRI were tested in a $9.4 \mathrm{~T} \mathrm{NMR}$ system.

\section{Experimental Section}

2.1. Materials. Acrylamide, ammonium persulfate, ferrous (III) chloride, ferric (II) chloride, TEMED $\left(N, N, N^{\prime}, N^{\prime}\right.$-tetramethylethylenediamine), tris(hydroxymethyl)aminomethane, and MTS (3-(4,5-dimethylthiazol-2-yl)-5-(3-carboxymethoxyphenyl)-2-(4-sulfophenyl)-2H-tetrazolium) were purchased from Sigma Aldrich (St. Louis, MO, USA). Isotonic saline solution $0.9 \%$ was from B. Braun (Melsungen, Germany). Ammonium, acetone, boric acid, and hydrochloric acid were from Penta (Prague, Czech Republic). The human Caucasian kidney carcinoma Caki-2 cell line (ATCC HTB-47) was obtained from the European Collection of Cell Cultures. Dulbecco's modified Eagle medium (DMEM), supplemented with fetal bovine serum, streptomycin, nonessential amino acids, L-glutamine, and sodium pyruvate, was purchased from Invitrogen (Gibco, Carlsbad, CA, USA).

\subsection{Preparation of Iron Oxide Nanoparticles. The synthesis} was carried out in water under ambient conditions. Ferrous and ferric chlorides were dissolved in $2 \mathrm{M} \mathrm{HCl}$ in molar ratio $2: 1$ and final concentration of $250 \mathrm{mM}$. Citrate ions were used as nucleation stabilisers. The reactor was filled with inert argon atmosphere and the NPs were slowly precipitated by dropping of $\mathrm{NH}_{3} \cdot \mathrm{H}_{2} \mathrm{O}(5.5 \mathrm{M})$ under vigorous mixing. The critical point of the synthesis is the core formation of the IOMPs and consequent crystal growth; therefore, the reaction conditions, including time and rate of base addition, were carefully optimised. The slow formation of nanoparticle seeds was followed by a faster formation of cores and a slow formation of shells stabilised by citrate ions for the whole time. The brownish-red product was precipitated with acetone, centrifuged for 5 minutes at 4,000 rpm, and the pellet 
dispersed in argon-bubbled water. These steps were repeated twice to reliably wash the NPs. After the last dispersion in water, the residues of acetone were evaporated under vacuum. The final product was sterilised by filtration through a $0.22 \mu \mathrm{m}$ filter. The NPs can be stored either in the lyophilised form or in an aqueous solution for a long time.

2.3. Characterisation Using AFM. Atomic force microscopy (AFM) images were captured on Ntegra Vita/Solaris (NTMDT, Russia) using the semicontact mode. The NPs were diluted in deionised water and $20 \mu \mathrm{L}$ of this dispersion was put on freshly cleaved mica and the water evaporated. As a probe, silicon cantilevers (AppNano, USA) with resonance frequency of $f_{0}=300 \mathrm{kHz}$, spring constant of $k=37 \mathrm{~N} / \mathrm{m}$ and a tip radius less than $10 \mathrm{~nm}$ were used. The scan rate was set at $1 \mathrm{~Hz}$. The images were flattened and processed for better quality and the particle sizes were determined from the height scans by means of Nova software.

2.4. Size Distribution. The particle size distribution of NPs was obtained by dynamic light scattering using Zetasizer Nano ZS (Malvern Instruments, UK). In the backscattering mode (angle of detection $173^{\circ}$, measuring position $1 \mathrm{~mm}$ from the wall of the cuvette), the instrument detects the time development of the intensity of light scattered by moving particles in the sample after irradiation with a $\mathrm{He}-$ Ne laser beam $(5 \mathrm{~mW}, 633 \mathrm{~nm})$. The data obtained undergo autocorrelation analysis and are subsequently evaluated using the methods of statistical data analysis (both Cumulant analysis and CONTIN algorithm) to obtain the particle size distributions. More details about these mathematical concepts can be found elsewhere [28]. The data shown in this work represent the mean distributions calculated from four independent repetitions. The measurement was carried out at laboratory temperature.

2.5. Zeta Potential. The zeta potential of the samples was determined using the method of electrophoretic light scattering using Zetasizer Nano ZS instrument (Malvern Instruments, UK). The instrument detects the Doppler shift between the laser beam $(5 \mathrm{~mW}, 633 \mathrm{~nm})$ passing through the cuvette with the sample and the reference beam passing outside the cuvette. The data are evaluated using phase analysis light scattering. A zeta dip cell was used to introduce an electric field into the sample (applied voltage $4.96 \pm 0.05 \mathrm{~V}$ ). All presented zeta potentials are reported as the mean value of five independent measurements at laboratory temperature.

2.6. Isotachophoretic Separation. The isotachophoretic (ITP) separation was used for concentration and size-sorting of small-sized NPs. The vertical homemade apparatus was described elsewhere [29]. The movement of raw negatively charged IOMPs was established by a system of two buffers: the leading electrolyte (LEL; $100 \mathrm{mM}$ Tris, $\mathrm{pH}$ 8.38) and the terminating electrolyte (TEL; $98 \mathrm{mM}$ Tris $+25 \mathrm{mM} \mathrm{H}_{3} \mathrm{BO}_{3}$ ). The separation channel of the ITP device was filled with $1 \%$ agarose gel prepared in LEL. The mixture of IOMPs $(140 \mu \mathrm{L}$; concentration $\left.45 \mathrm{mg} \cdot \mathrm{mL}^{-1}\right), 50 \%$ glycerol $(40 \mu \mathrm{L})$, and $10 \mathrm{x}$ concentrated TEL $(20 \mu \mathrm{L})$ was loaded on agarose gel. The ITP was performed in constant voltage mode at $50 \mathrm{~V}$. The higher concentration of IOMPs resulted in a total running time of $80 \mathrm{~min}$. The final band (width of $1 \mathrm{~cm}$ ) was cut to 4 pieces and IOMPs were transferred from the gel to $20 \mu \mathrm{L}$ of Tris buffer and PAGE electrophoresis of those separated IOMPs was performed in accordance with supporting information for [29].

2.7. Cytotoxicity Assay. The cell viability was tested by the MTS method. Caki-2 cells were harvested in 24-well plate at an initial density of $50 \times 10^{3}$ cells per well. The cell line was maintained in $500 \mu \mathrm{L}$ (per well) of DMEM supplemented with fetal bovine serum $(20 \%), 100 \mu \mathrm{g} \cdot \mathrm{mL}^{-1}$ streptomycin, $0.1 \mathrm{mM}$ nonessential amino acids, $2 \mathrm{mM}$ L-glutamine, and $1 \mathrm{mM}$ sodium pyruvate. The incubation conditions were $37^{\circ} \mathrm{C}$ with $5 \% \mathrm{CO}_{2}$. Subsequently, after 24 hours of incubation, NPs in four different concentrations $\left(50,20\right.$, and $5 \mu \mathrm{g} \cdot \mathrm{mL}^{-1}$, with $0 \mu \mathrm{g} \cdot \mathrm{mL}^{-1}$ as a negative control) were added to the cells and the plates were incubated for $6,24,48$, and $72 \mathrm{~h}$. To each well, $50 \mu \mathrm{L}$ of $5 \mathrm{mg} \cdot \mathrm{mL}^{-1}$ MTS solution in PBS was added at $6,24,48$, and $72 \mathrm{~h}$ after NP addition and the plates were incubated for $1 \mathrm{~h}$ at $37^{\circ} \mathrm{C}$. Absorbance was measured using the Multi-Detection Microplate Reader (BioTek, Winooski, VT, USA) at a wavelength of $490 \mathrm{~nm}$. Cell viability was calculated from $\mathrm{OD}_{490}$ value of the experimental group (in triplicate) by subtracting that of the blank group.

2.8. Relaxivity and MRI Studies. All experiments were performed using a 9.4 T NMR system (Bruker-BioSpec 94/ 30 USR, Ettlingen, Germany). The series with different concentrations of IOMPs $(0,0.08,0.17,0.29,0.42$, and $0.62 \mathrm{mM}$ of $\left.\gamma-\mathrm{Fe}_{2} \mathrm{O}_{3}\right)$ in saline solutions containing $1.5 \%$ agar gel were prepared for relaxivity MRI phantom studies. Agar gel was preferred to a simple aqueous solution in order to avoid inhomogeneity due to possible sedimentation and a remaining aggregation. Images for $T_{1}$ and $T_{2}$ quantification were acquired using the Rapid Acquisition with Relaxation Enhancement (RARE) pulse sequence [30]. The parameters for $T_{1}$ quantification were $\mathrm{TR}=50,100,250,500,1000$, 2000, 3000, 5000, 10000, and $15000 \mathrm{~ms}$; TE = $10 \mathrm{~ms}$; for $T_{2}$ quantification were $\mathrm{TR}=15,000 \mathrm{~ms} ; \mathrm{TE}=10,30,50,70,90$, 110,130 , and $150 \mathrm{~ms}$, where TR is the repetition time and TE the effective echo time. Slice thickness (SL) for all acquisitions was $1 \mathrm{~mm}$, matrix $128 \times 128$, field of view $(\mathrm{FOV}) 6 \times 4 \mathrm{~cm}$, single slice, and RARE factor $=2$. The values of $T_{1}$ and $T_{2}$ were calculated for each sample in manually drawn regions of interest (ROIs) using the Image Sequence Analysis tool (ParaVision v.5.1, Bruker BioSpin, Ettlingen, Germany). $r_{1}$ and $r_{2}$ relaxivities were calculated as the proportionality constants of the linear relation between the reciprocal relaxation time and $\gamma-\mathrm{Fe}_{2} \mathrm{O}_{3}$ concentration (in IOMP NPs). The samples were measured at room temperature and at $37.7^{\circ} \mathrm{C}$.

\section{Results and Discussion}

3.1. Characterisation of Citrate-Stabilised IOMPs. Using the chemical coprecipitation technique, well-dispersible IOMPs 

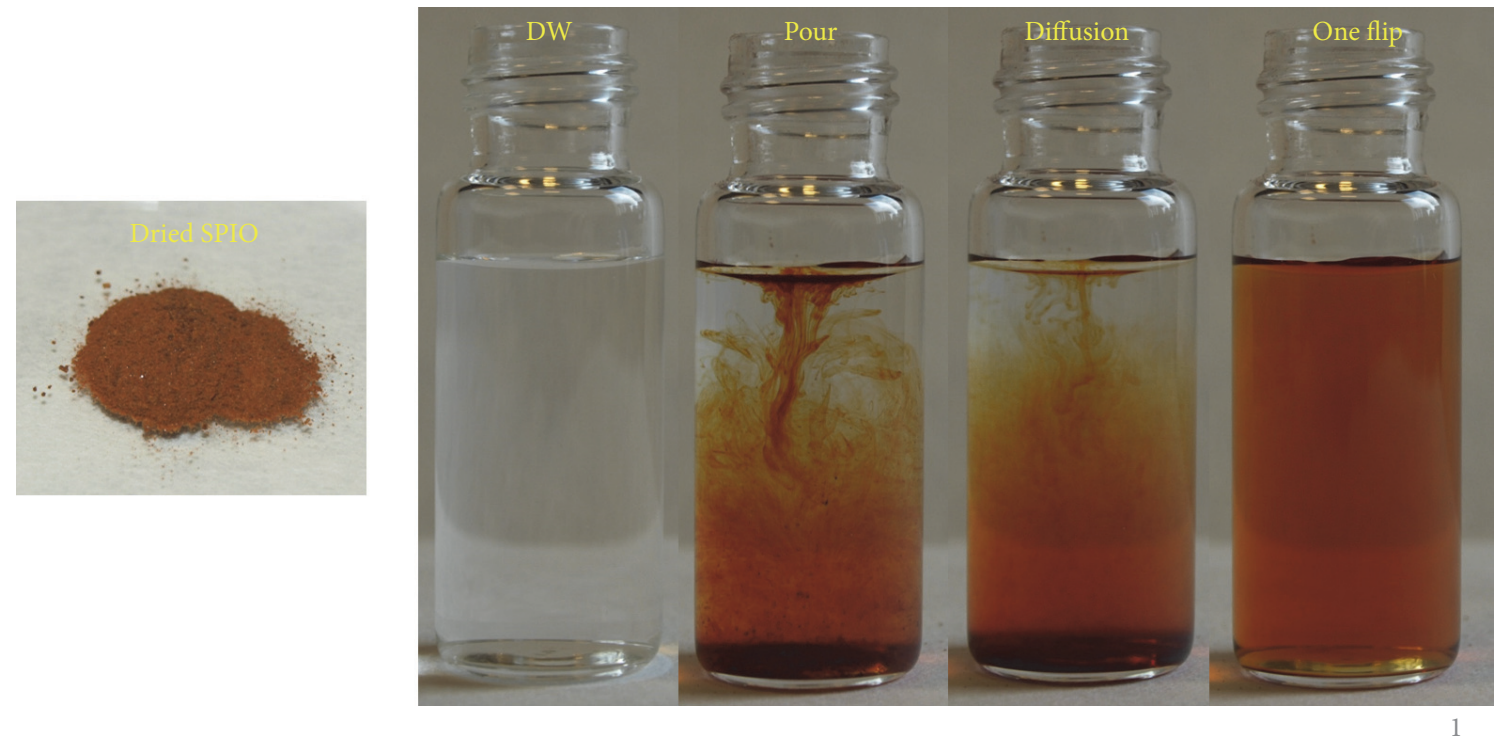

FIGURE 1: Demonstration of very fast reconstitution of dried IOMP nanoparticles in water. No special conditions (sonication, detergents, etc.) are needed. Only adding IOMPs in water and one flip of the vial is sufficient for stable dispersion in a few seconds.

were prepared. In the presence of oxygen, magnetite $\left(\mathrm{Fe}_{3} \mathrm{O}_{4}\right)$ is thermodynamically unstable and it is highly sensitive to oxidation into maghemite $\left(\gamma-\mathrm{Fe}_{2} \mathrm{O}_{3}\right)$. For smaller NPs a higher rate of oxidation of magnetite to maghemite should be expected. Therefore, the coprecipitation method is usually limited by a broad distribution of NP sizes. Here, the reaction conditions were carefully optimised to overcome this disadvantage. A slower reaction during the preparation results in very fine NPs that are highly stable in water for long periods. Furthermore, no special conditions were needed to form stable brown suspensions in water. Dispersion of lyophilised nanoparticles was very fast, in the order of few seconds (Figure 1).

Particle size was determined by atomic force microscopy. The average size derived from the height scan was below $2 \mathrm{~nm}$. The distribution profile of sizes from the AFM height scan is shown in Figure 2(a). A section of the AFM scan is included in the inset. The results from the AFM correspond to the distribution acquired from DLS (Figure 2(b)). The hydrodynamic diameter distribution of the IOMPs was of $3.6 \mathrm{~nm}$ and volume ratio of $16.9 \%$. The hydrodynamic diameter by DLS is slightly larger than that determined by AFM, as expected. The DLS and AFM analyses conducted on standard polystyrene NPs have been compared previously and small differences between both methods and nominal values were deduced [31]. A similar study has been performed elsewhere and it was observed that DLS was limited for accurate particle sizing and mostly DLS provides higher values [32]. Figure 2 indicates that the optimised coprecipitation method effectively produces a well-dispersed and narrower distribution of particle diameters. The high stability of IOMP NPs is given by their very low electrostatic potential. As can be seen by the measurement of zeta potential, the citrate-stabilised IOMP NPs are very stable (Table 1). The zeta potential of NPs was constant at around $-39 \mathrm{mV}$ for long-term storage (over 16 months).
TABLE 1: Stability of zeta potential of IOMPs nanoparticles during two months.

\begin{tabular}{lcc}
\hline Time & $\zeta$ Potential $[\mathrm{mV}]$ & $\mathrm{SD}[\mathrm{mV}]$ \\
\hline 0 days & -39.5 & 1.0 \\
4 days & -39.1 & 3.0 \\
9 days & -37.8 & 0.9 \\
11 days & -39.3 & 2.5 \\
14 days & -39.2 & 1.6 \\
60 days & -39.2 & 1.7 \\
\hline
\end{tabular}

In addition, there was neither macroscopic nor microscopic significant change in behaviour and stability of NPs after 16-month storage in dispersion at room temperature, even at higher concentrations. For in vivo applications, the NPs have to be sterile. Autoclaving was performed for 20 minutes at $121^{\circ} \mathrm{C}$ without any impact on the IOMPs' properties. A combination of acetone precipitation during the washing process and filtration through a $0.22 \mu \mathrm{m}$ filter is also sufficient and has no effect on the stability of NPs.

3.2. Isotachophoretic Separation. For meaningful MR imaging, it is necessary to use small as well as uniformly magnetic NPs with well-defined sizes. Different separation techniques are currently available for size- and shape-selective purification, such as size exclusion chromatography (SEC) [33], density gradient centrifugation [34], magnetic separation [35], and electrophoretic methods [36]. Relatively low capacity and dilution of samples are the disadvantages of the SEC method. The centrifugation and magnetic separation methods are limited by the stability of dispersion. The high stability of our NPs allows neither centrifugation nor magnetic separation. The isotachophoresis was chosen here as the efficient technique for the separation of highly stable 


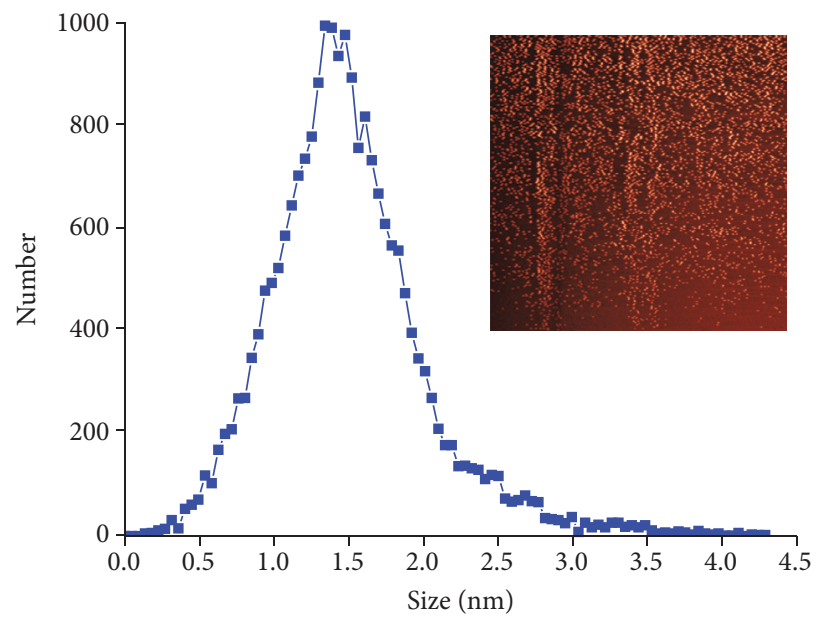

(a)

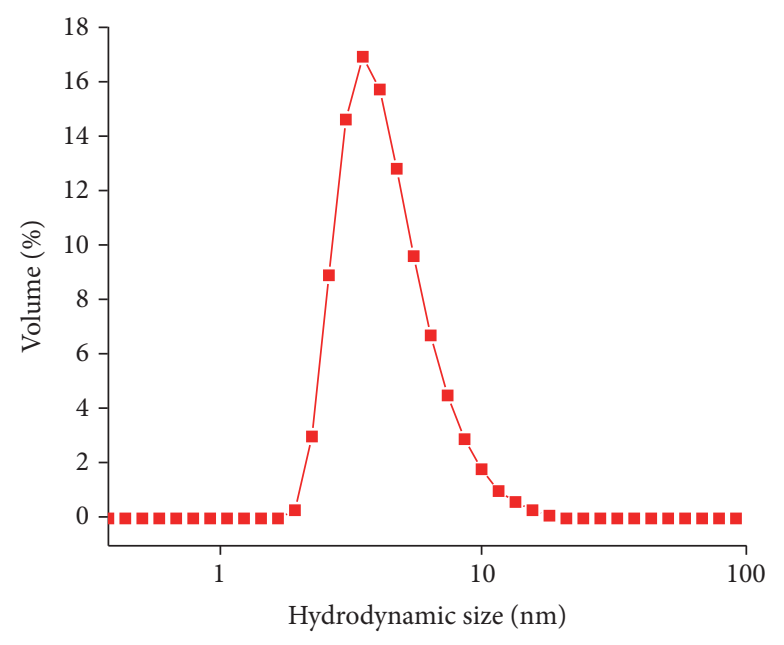

(b)

Figure 2: AFM (a) and DLS (b) size profiles. The histogram of IOMP sizes derived from height profile of AFM scan on square $2 \times 4 \mu \mathrm{m}$. The section of AFM scan (inset) represents the $2 \times 2 \mu \mathrm{m}$ area. The DLS hydrodynamic size distribution by volume shows narrower peak corresponding to AFM height profile.

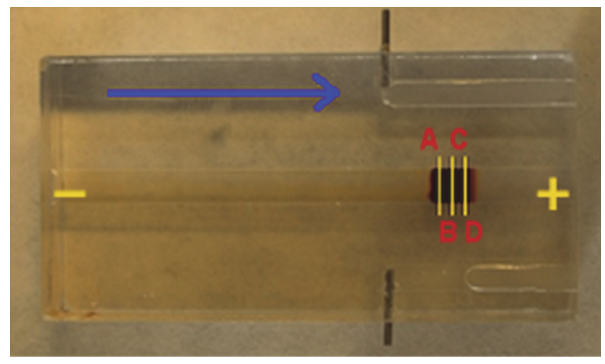

(a)

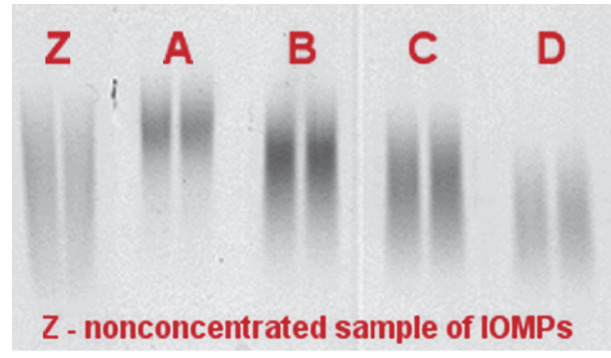

(b)

FIGURE 3: Isotachophoretic size sorting and preconcentration (a) with electrophoretic characterisation of IOMP NPs (b). The ITP cell was filled with agarose gel (a). The potential of electrodes and the sample movement during preconcentration is depicted. Individual zones (A, B, $\mathrm{C}$, and D) of the cut concentrated sample were electrophoretically separated on the PAGE and nonconcentrated IOMPs sample was used as control (Z). The diffusion of concentrated IOMPs in the gel is very fast as can be seen on unfocused bars.

NPs. The PAGE electrophoresis (Figure 3) of separated fractions revealed narrower size distribution and quality of the developed synthetic process. The distribution and intensity of bars correspond to the results achieved by DLS and AFM. In the electrophoregram, the " $\mathrm{B}$ " band represents the major size component contained in the original sample. Besides the sizesorting effect, the concentration of various individual sizes is a valuable advantage of isotachophoresis. In this case, the sample was 10x more concentrated than the nonseparated sample applied on the column. In addition, there was no significant instability of NPs during electrophoretic movements. The NPs were free of aggregates and retained good dispersibility.

3.3. Cytotoxicity Assay. To evaluate the potential cytotoxicity of NPs, a cell viability test (MTS assay) was performed. The MTS assay is a colorimetric method based on the reduction of MTS tetrazolium compound by viable cells. The product of the reaction is a purple-coloured formazan soluble in the DMEM media. The conversion is performed due to $\mathrm{NAD}(\mathrm{P}) \mathrm{H}$-dependent dehydrogenase enzymes in metabolically active cells. The formazan dye produced by viable cells is subsequently quantified by measuring the absorbance at $490 \mathrm{~nm}$ (Figure 4). It was observed that the highest concentration of NPs $\left(50 \mu \mathrm{g} \cdot \mathrm{mL}^{-1}\right)$ caused a shock to cells during first six hours after NP addition. However, after this initial shock, at the following time steps, the cells grew in the same manner as cells with lower NP loadings or as the negative control. These data show that even the highest tested NP concentration did not exhibit toxicity in the Caki- 2 cell.

3.4. Relaxivity and MRI Studies. For the IOMPs measured in $1.5 \%$ agar gel at $9.4 \mathrm{~T}$, the relaxivities $r_{1}$ were $0.25 \mathrm{mM}^{-1} \mathrm{Fe}_{2} \mathrm{O}_{3} \mathrm{~s}^{-1}$ at room temperature and $0.24 \mathrm{mM}^{-1} \mathrm{Fe}_{2} \mathrm{O}_{3} \mathrm{~s}^{-1}$ at $37.7^{\circ} \mathrm{C}$. The main reason for the low values was most probably the high magnetic field applied here $(9.4 \mathrm{~T})$, compared to the magnetic field usually used in the most publications (1.5 and $3 \mathrm{~T})$. The relaxivities 


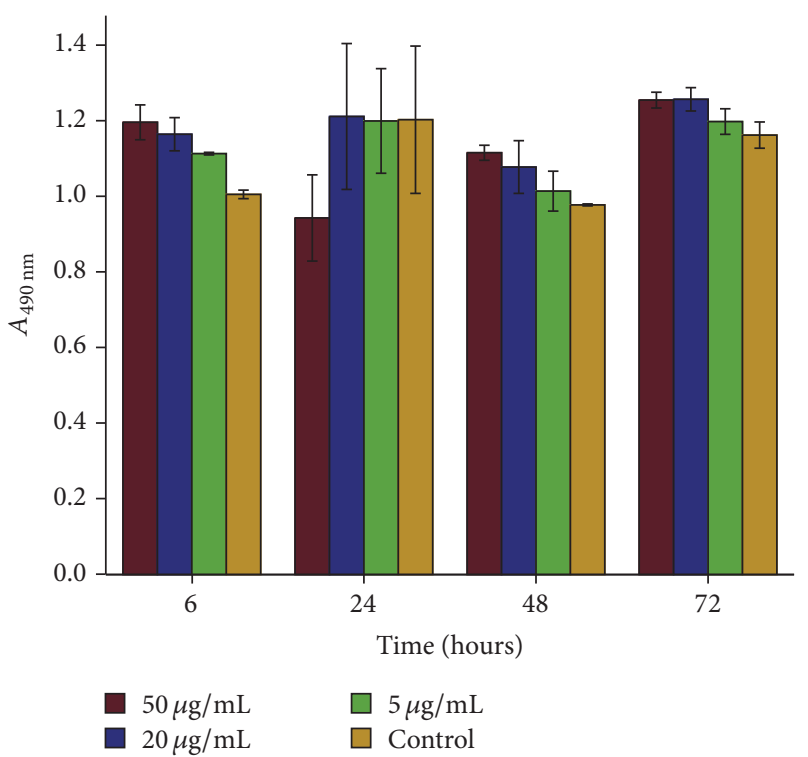

FIgure 4: Cytotoxicity of IOMP NPs on Caki-2 cells. Three different concentrations of IOMPs were added to the Caki-2 cells and incubated for $6,24,48$, and 72 hours. The cytotoxicity has been compared with the negative control with 0 concentrations of NPs. At 24 hours, the toxic shock by adding of $50 \mu \mathrm{g} \cdot \mathrm{mL}^{-1}$ can be seen. A couple of hours later, proliferative behaviour was influenced even by the lowest concentration of added IOMPs.

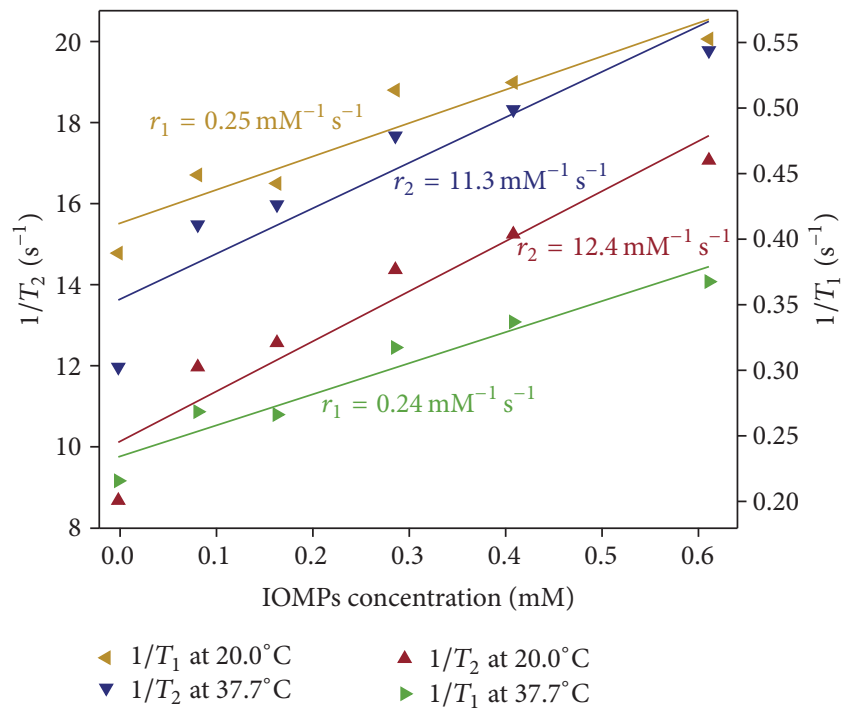

FIGURE 5: Plot of the proton relaxation rates $\left(1 / T_{2}\right.$ and $\left.1 / T_{1}\right)$ of IOMPs. The concentration gradient of NPs was prepared in saline containing $1.5 \%$ agarose. The relaxation times were measured at $20^{\circ} \mathrm{C}$ and $37.7^{\circ} \mathrm{C}$. The calculated relaxivities are shown in the figure.

$r_{2}$ were $12.4 \mathrm{mM}^{-1} \mathrm{Fe}_{2} \mathrm{O}_{3} \mathrm{~s}^{-1}$ at room temperature and $11.3 \mathrm{mM}^{-1} \mathrm{Fe}_{2} \mathrm{O}_{3} \mathrm{~s}^{-1}$ at $37.7^{\circ} \mathrm{C}$, respectively. All data, including the relaxivities, are shown in Figure 5. These relaxivity values correspond to values claimed on commercial NPs with a nominal diameter of $20 \mathrm{~nm}$ [37]. In their study, Najafian et al. deduced that there is an influence of the physicochemical properties of the superparamagnetic NPs

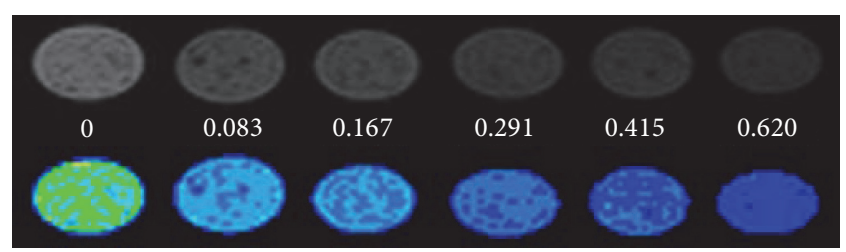

FIGURE 6: Phantom images of concentration gradient of IOMPs. $\mathrm{T}_{2}$ weighted RARE sequence measured in saline containing $1.5 \%$ agarose: TR $15 \mathrm{~s}$; TE $110 \mathrm{~ms}$ (negative contrast). The concentration of IOMPs is indicated in $\mathrm{mM}$. Identical phantoms are shown in black and white (upper row) and pseudocolour (lower row) scale.

attributable to different surface modifications. The surface charge seems to affect relaxivity more than the hydrodynamic size of NPs. $r_{2} / r_{1}$ ratio, which was $\sim 47$ for our NPs at $37.7^{\circ} \mathrm{C}$ and $9.4 \mathrm{~T}$, indicates a possible use of these NPs as a $T_{2^{-}}$ contrast agent.

The MRI images of the phantom with different concentrations of IOMPs are shown in Figure 6. The relaxivities of IOMPs were regularly measured within one month and showed high stability and no aggregation in a strong magnetic field (data not shown). The data confirm that our ultrasmall NPs can serve as alternative $T_{2}$ MRI contrast agents. Due to their very small size and good hydrophilicity, the produced NPs should easily permeate tissues and achieve more widespread tissue distribution in a short time.

\section{Conclusions}

Well-dispersible, ultra-small, and hydrophilic iron oxide nanoparticles were synthesised by a chemical coprecipitation method. A citrate shell stabilises the dispersion and protects NPs against aggregation and sedimentation. The procedure was optimised for the production of NPs with a narrow size distribution, which was confirmed by AFM and DLS measurements. High stability was confirmed by monitoring of zeta potential, which detected no change over several months. Isotachophoresis (ITP) was used for preconcentration of NPs. It was clearly shown that ITP could also be applied for purification and sorting of IOMPs. In vitro cytotoxicity MTS assay confirmed biocompatibility of the prepared NPs. The relaxivity values were determined in a 9.4 T magnetic field. $r_{2}$ value and $r_{2} / r_{1}$ ratio suggest that our NPs can be potentially used as $T_{2}$ negative contrast probes.

\section{Abbreviations}

AFM: Atomic force microscopy

DLS: Dynamic light scattering

IOMPs: Iron oxide magnetic particles

ITP: Isotachophoresis

LEL: Leading electrolyte

MRI: Magnetic resonance imaging

NMR: Nuclear magnetic resonance

NPs: Nanoparticles

SPIOs: Superparamagnetic iron oxide nanoparticles

TEL: Terminating electrolyte 
TE: Effective echo time

TR: Repetition time.

\section{Competing Interests}

The authors declare that they have no competing interests.

\section{Authors' Contributions}

David Kovár synthesised, modified, and characterised nanoparticles by AFM. Aneta Malá, Radovan Jiřík, and Zenon Starčuk Jr. performed and analysed MRI acquisitions. Jitka Mlčochová and Ondřej Slabý carried out the cell cytotoxicity study. Michal Kalina and Zdenka Fohlerová carried out and participated in DLS and zeta potential measurements. Antonín Hlaváček, Zdenka Fohlerová, and Petr Skládal participated in the design of the ITP separation technique. Jaromír Hubálek participated in design of study and coordinated to draft the manuscript. All authors read and approved the final manuscript.

\section{Acknowledgments}

The work has been supported by the Ministry of Education, Youth, and Sports of the Czech Republic under the Project CEITEC 2020 (LQ1601) and Projects LO1212 and LO1211 and by Grant Project GACR 16-18257S.

\section{References}

[1] H. B. Na, I. C. Song, and T. Hyeon, "Inorganic nanoparticles for MRI contrast agents," Advanced Materials, vol. 21, no. 21, pp. 2133-2148, 2009.

[2] J. Y. Park, M. J. Baek, E. S. Choi et al., "Paramagnetic ultrasmall gadolinium oxide nanoparticles as advanced T 1 MRI contrast agent: account for large longitudinal relaxivity, optimal particle diameter, and in Vivo T1 MR images," ACS Nano, vol. 3, no. 11, pp. 3663-3669, 2009.

[3] F. Hu and Y. S. Zhao, "Inorganic nanoparticle-based T1 and T1/T2 magnetic resonance contrast probes," Nanoscale, vol. 4, no. 20, pp. 6235-6243, 2012.

[4] F. Hu, K. W. MacRenaris, E. A. Waters et al., "Ultrasmall, water-soluble magnetite nanoparticles with high relaxivity for magnetic resonance imaging," The Journal of Physical Chemistry C, vol. 113, no. 49, pp. 20855-20860, 2009.

[5] M. Rohrer, H. Bauer, J. Mintorovitch, M. Requardt, and H.J. Weinmann, "Comparison of magnetic properties of MRI contrast media solutions at different magnetic field strengths," Investigative Radiology, vol. 40, no. 11, pp. 715-724, 2005.

[6] D. Frascione, C. Diwoky, G. Almer et al., "Ultrasmall superparamagnetic iron oxide (USPIO)-based liposomes as magnetic resonance imaging probes," International Journal of Nanomedicine, vol. 7, pp. 2349-2359, 2012.

[7] R. Qiao, C. Yang, and M. Gao, "Superparamagnetic iron oxide nanoparticles: from preparations to in vivo MRI applications," Journal of Materials Chemistry, vol. 19, no. 35, pp. 6274-6293, 2009.

[8] C. F. G. C. Geraldes and S. Laurent, "Classification and basic properties of contrast agents for magnetic resonance imaging,"
Contrast Media and Molecular Imaging, vol. 4, no. 1, pp. 1-23, 2009.

[9] A. J. L. Villaraza, A. Bumb, and M. W. Brechbiel, "Macromolecules, dendrimers, and nanomaterials in magnetic resonance imaging: the interplay between size, function, and pharmacokinetics," Chemical Reviews, vol. 110, no. 5, pp. 29212959, 2010.

[10] G. Huang, H. Chen, Y. Dong et al., "Superparamagnetic iron oxide nanoparticles: amplifying ros stress to improve anticancer drug efficacy," Theranostics, vol. 3, no. 2, pp. 116-126, 2013.

[11] C. Jiang, J. Jia, and S. Zhai, "Mechanistic understanding of toxicity from nanocatalysts," International Journal of Molecular Sciences, vol. 15, no. 8, pp. 13967-13992, 2014.

[12] L. Tong, M. Zhao, S. Zhu, and J. Chen, "Synthesis and application of superparamagnetic iron oxide nanoparticles in targeted therapy and imaging of cancer," Frontiers of Medicine, vol. 5, no. 4, pp. 379-387, 2011.

[13] T. Sugimoto and E. Matijević, "Formation of uniform spherical magnetite particles by crystallization from ferrous hydroxide gels," Journal of Colloid and Interface Science, vol. 74, no. 1, pp. 227-243, 1980.

[14] R. Massart, "Preparation of aqueous magnetic liquids in alkaline and acidic media," IEEE Transactions on Magnetics, vol. 17, no. 2, pp. 1247-1248, 1981.

[15] A. Bee, R. Massart, and S. Neveu, "Synthesis of very fine maghemite particles," Journal of Magnetism and Magnetic Materials, vol. 149, no. 1-2, pp. 6-9, 1995.

[16] S. Laurent, D. Forge, M. Port et al., "Magnetic iron oxide nanoparticles: synthesis, stabilization, vectorization, physicochemical characterizations, and biological applications," Chemical Reviews, vol. 108, no. 6, pp. 2064-2110, 2008.

[17] M. Safdarian, P. Hashemi, and M. Adeli, "One-step synthesis of agarose coated magnetic nanoparticles and their application in the solid phase extraction of $\mathrm{Pd}(\mathrm{II})$ using a new magnetic field agitation device," Analytica Chimica Acta, vol. 774, pp. 44-50, 2013.

[18] C. Tassa, S. Y. Shaw, and R. Weissleder, "Dextran-coated iron oxide nanoparticles: a versatile platform for targeted molecular imaging, molecular diagnostics, and therapy," Accounts of Chemical Research, vol. 44, no. 10, pp. 842-852, 2011.

[19] R. G. López, M. G. Pineda, G. Hurtado et al., "Chitosancoated magnetic nanoparticles prepared in one step by reverse microemulsion precipitation," International Journal of Molecular Sciences, vol. 14, no. 10, pp. 19636-19650, 2013.

[20] H. Yilmaz and S. H. Sanlier, "Preparation of magnetic gelatin nanoparticles and investigating the possible use as chemotherapeutic agent," Artificial Cells, Nanomedicine and Biotechnology, vol. 41, no. 2, pp. 69-77, 2013.

[21] M. M. Yallapu, S. P. Foy, T. K. Jain, and V. Labhasetwar, "PEG-functionalized magnetic nanoparticles for drug delivery and magnetic resonance imaging applications," Pharmaceutical Research, vol. 27, no. 11, pp. 2283-2295, 2010.

[22] A. Petri-Fink, B. Steitz, A. Finka, J. Salaklang, and H. Hofmann, "Effect of cell media on polymer coated superparamagnetic iron oxide nanoparticles (SPIONs): colloidal stability, cytotoxicity, and cellular uptake studies," European Journal of Pharmaceutics and Biopharmaceutics, vol. 68, no. 1, pp. 129-137, 2008.

[23] L. P. Ramírez and K. Landfester, "Magnetic polystyrene nanoparticles with a high magnetite content obtained by miniemulsion processes," Macromolecular Chemistry and Physics, vol. 204, no. 1, pp. 22-31, 2003. 
[24] T. Linemann, L. B. Thomsen, K. G. Du Jardin et al., "Development of a novel lipophilic, magnetic nanoparticle for in vivo drug delivery," Pharmaceutics, vol. 5, no. 2, pp. 246-260, 2013.

[25] S. Gyergyek, M. Drofenik, and D. Makovec, "Oleic-acid-coated $\mathrm{CoFe}_{2} \mathrm{O}_{4}$ nanoparticles synthesized by co-precipitation and hydrothermal synthesis," Materials Chemistry and Physics, vol. 133, no. 1, pp. 515-522, 2012.

[26] M. Iijima, Y. Yonemochi, M. Kimata, M. Hasegawa, M. Tsukada, and H. Kamiya, "Preparation of agglomeration-free hematite particles coated with silica and their reduction behavior in hydrogen," Journal of Colloid and Interface Science, vol. 287, no. 2, pp. 526-533, 2005.

[27] M. Kosmulski, "Positive electrokinetic charge of silica in the presence of chlorides," Journal of Colloid and Interface Science, vol. 208, no. 2, pp. 543-545, 1998.

[28] R. Xu, Particle Characterization: Light Scattering Methods, Springer, Dordrecht, The Netherlands, 2006.

[29] A. Hlavacek and P. Skládal, "Isotachophoretic purification of nanoparticles: tuning optical properties of quantum dots," Electrophoresis, vol. 33, no. 9-10, pp. 1427-1430, 2012.

[30] J. Hennig, A. Nauerth, and H. Friedburg, "RARE imaging: a fast imaging method for clinical MR," Magnetic Resonance in Medicine, vol. 3, no. 6, pp. 823-833, 1986.

[31] C. M. Hoo, N. Starostin, P. West, and M. L. Mecartney, "A comparison of atomic force microscopy (AFM) and dynamic light scattering (DLS) methods to characterize nanoparticle size distributions," Journal of Nanoparticle Research, vol. 10, no. 1, pp. 89-96, 2008.

[32] M. Baalousha and J. R. Lead, "Rationalizing nanomaterial sizes measured by atomic force microscopy, flow field-flow fractionation, and dynamic light scattering: sample preparation, polydispersity, and particle structure," Environmental Science and Technology, vol. 46, no. 11, pp. 6134-6142, 2012.

[33] T. Arita, T. Yoshimura, and T. Adschiri, "Size exclusion chromatography of quantum dots by utilizing nanoparticle repelling surface of concentrated polymer brush," Nanoscale, vol. 2, no. 8 , pp. 1467-1473, 2010.

[34] B. Xiong, J. Cheng, Y. Qiao, R. Zhou, Y. He, and E. S. Yeung, "Separation of nanorods by density gradient centrifugation," Journal of Chromatography A, vol. 1218, no. 25, pp. 3823-3829, 2011.

[35] G. D. Moeser, K. A. Roach, W. H. Green, T. Alan Hatton, and P. E. Laibinis, "High-gradient magnetic separation of coated magnetic nanoparticles," AIChE Journal, vol. 50, no. 11, pp. 2835-2848, 2004.

[36] A. I. López-Lorente, B. M. Simonet, and M. Valcárcel, "Electrophoretic methods for the analysis of nanoparticles," Trends in Analytical Chemistry, vol. 30, no. 1, pp. 58-71, 2011.

[37] N. Najafian, S. Shanehsazzadeh, F. Hajesmaeelzadeh, A. Lahooti, C. Gruettner, and M. A. Oghabian, "Effect of functional group and surface charge of PEG and dextran-coated USPIO as a contrast agent in MRI on relaxivity constant," Applied Magnetic Resonance, vol. 46, no. 6, pp. 685-692, 2015. 

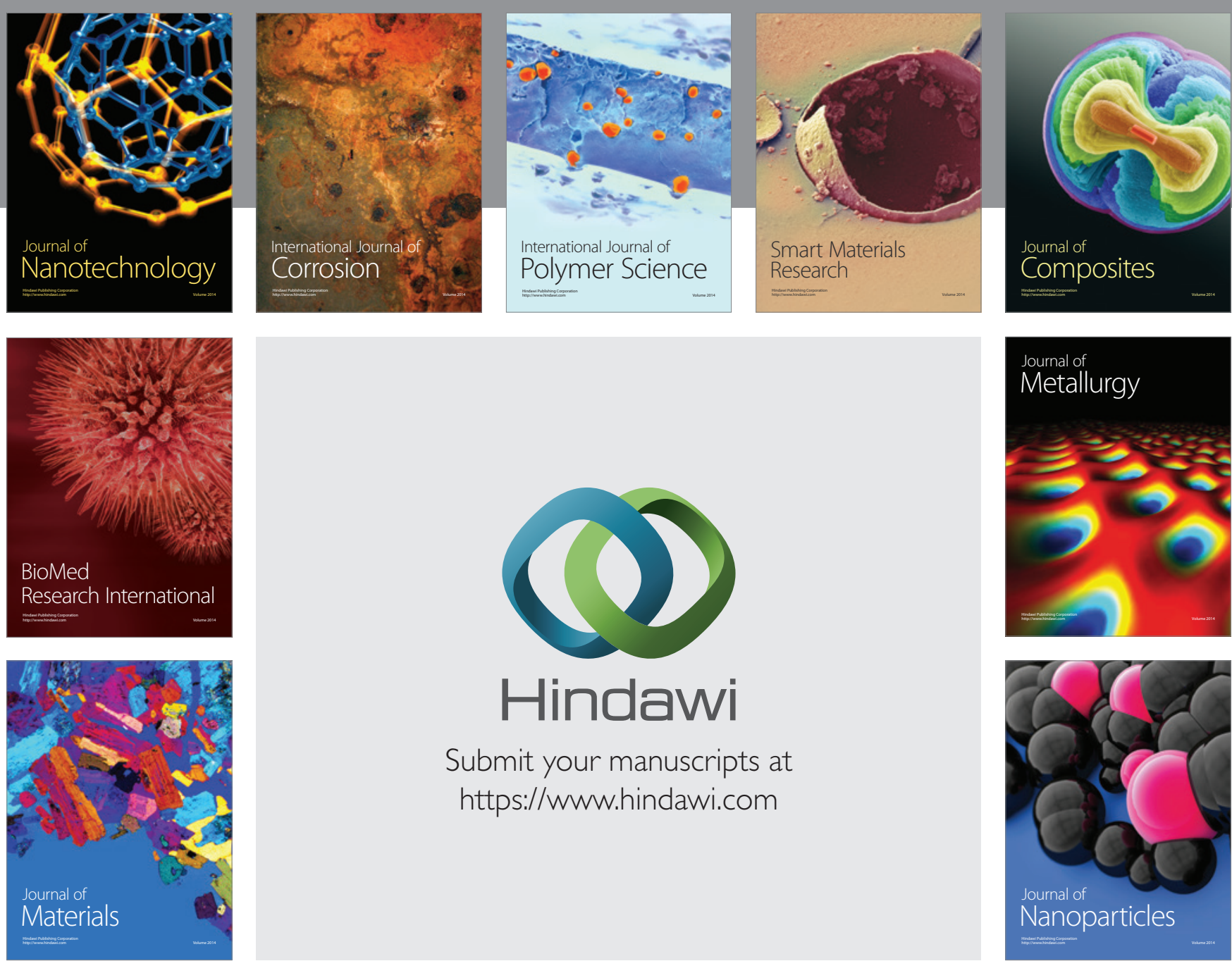

\section{Hindawi}

Submit your manuscripts at

https://www.hindawi.com

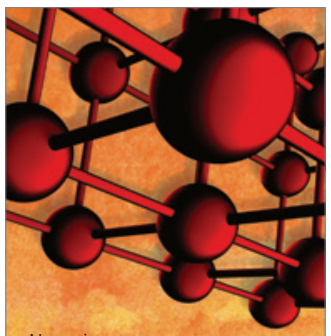

Materials Science and Engineering
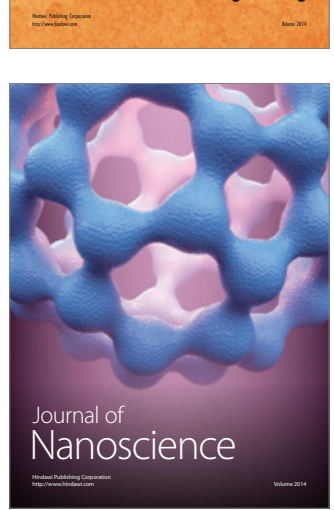
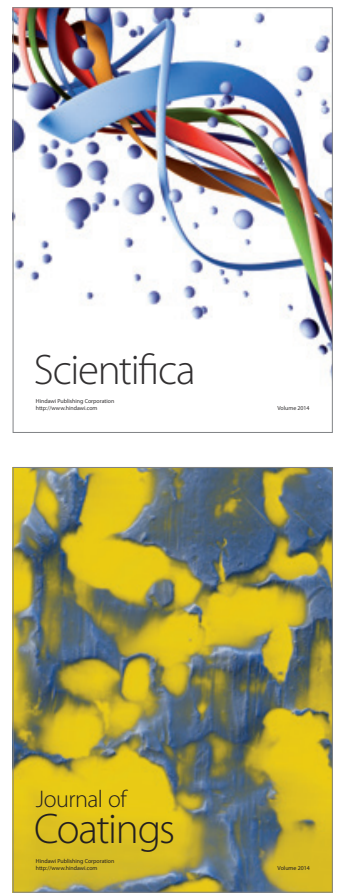
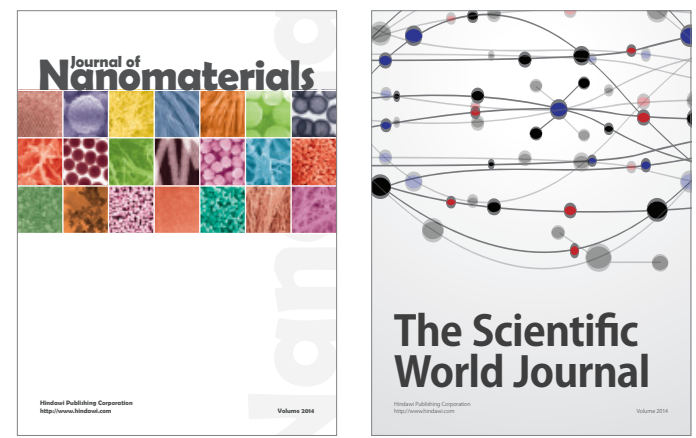

The Scientific World Journal
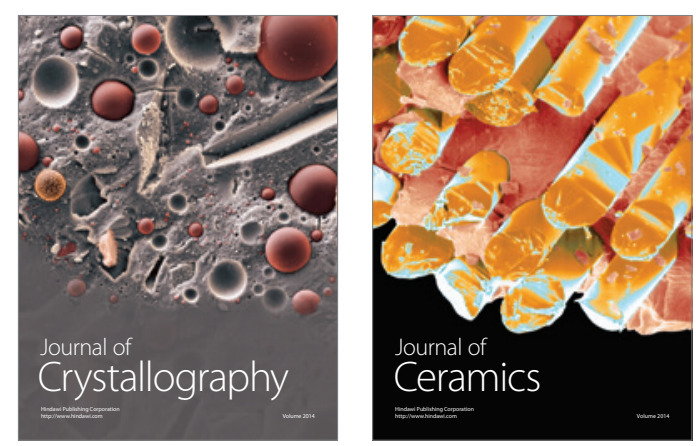
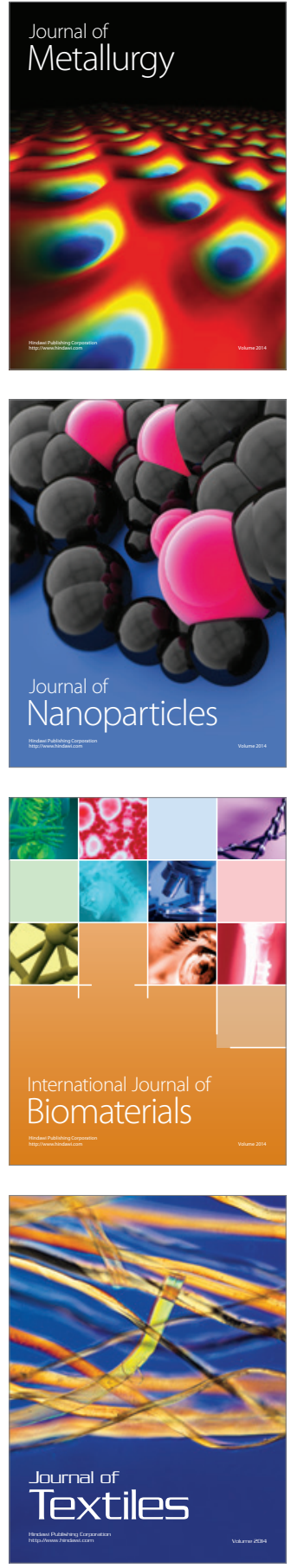